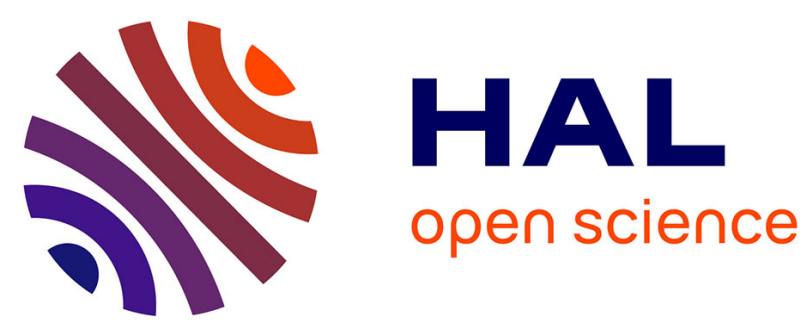

\title{
Threshold for a Reduction in Anticholinergic Burden to Decrease Behavioral and Psychological Symptoms of Dementia
}

Yacine Jaïdi, Adrien Guilloteau, Vignon Nonnonhou, Laurie-Anne Bertholon, Sarah Badr, Isabella Morrone, Jean-Luc Novella, Rachid Mahmoudi

\section{To cite this version:}

Yacine Jaïdi, Adrien Guilloteau, Vignon Nonnonhou, Laurie-Anne Bertholon, Sarah Badr, et al.. Threshold for a Reduction in Anticholinergic Burden to Decrease Behavioral and Psychological Symptoms of Dementia. Journal of the American Medical Directors Association, 2019, 20 (2), pp.159-164.e3. 10.1016/j.jamda.2018.10.015 . hal-02799691

\section{HAL Id: hal-02799691 \\ https://hal.science/hal-02799691}

Submitted on 21 Oct 2021

HAL is a multi-disciplinary open access archive for the deposit and dissemination of scientific research documents, whether they are published or not. The documents may come from teaching and research institutions in France or abroad, or from public or private research centers.
L'archive ouverte pluridisciplinaire HAL, est destinée au dépôt et à la diffusion de documents scientifiques de niveau recherche, publiés ou non, émanant des établissements d'enseignement et de recherche français ou étrangers, des laboratoires publics ou privés.

\section{(ㅇ)(1) $\$$}

Distributed under a Creative Commons Attribution - NonCommerciall 4.0 International 
Original Study

\title{
Threshold for a Reduction in Anticholinergic Burden to Decrease Behavioral and Psychological Symptoms of Dementia
}

\author{
Yacine Jaïdi MD, MSc ${ }^{\mathrm{a}, \mathrm{b}}$, Adrien Guilloteau MD, MSc ${ }^{\mathrm{c}, \mathrm{d}}$, Vignon Nonnonhou MD ${ }^{\mathrm{a}}$, \\ Laurie-Anne Bertholon MD ${ }^{a}$, Sarah Badr MD ${ }^{a}$, Isabella Morrone PhD ${ }^{\mathrm{a}, \mathrm{b}}$, \\ Jean-Luc Novella MD, PhD ${ }^{\mathrm{a}, \mathrm{b}}$, Rachid Mahmoudi MD, $\mathrm{PhD}^{\mathrm{a}, \mathrm{b}, *}$ \\ ${ }^{a}$ Department of Geriatrics and Internal Medicine, Reims University Hospitals, Maison Blanche Hospital, Reims, France \\ ${ }^{\mathrm{b}}$ Faculty of Medicine, EA 3797, University of Reims Champagne-Ardenne, Reims, France \\ ${ }^{\mathrm{c}}$ Epidemiology and infection control unit, University hospital of Dijon, Dijon, France \\ d INSERM U1231, EPICAD Team, Dijon, France
}

\section{Keywords:}

Cholinergic antagonist

dementia

behavioral disorders

drug effects

older

\begin{abstract}
A B S T R A C T
Background: A high anticholinergic burden $(\mathrm{AB})$ is associated with the occurrence of behavioral and psychological symptoms (BPSDs), which are frequent in dementia.

Objectives: Our aim was to determine the threshold for a reduction in $\mathrm{AB}$ that would lead to a clinically significant improvement in BPSDs (in terms of frequency, severity, and disruptiveness).

Design: A single-center prospective study.

Settings: Dedicated geriatric care unit specializing in the management of patients with dementia.

Participants: The study involved older patients with dementia, hospitalized for management of BPSDs. Methods: One hundred forty-seven patients were included (mean age $=84.1 \pm 5.2$ years). The AB was assessed using 3 scales, namely, the Anticholinergic Drug Scale (ADS), the Anticholinergic Cognitive Burden scale (ACB), and the Anticholinergic Risk Scale (ARS). A clinically significant improvement in BPSDs was defined as a reduction of 4 points in the frequency $\times$ severity $(F \times S)$ score of the Neuropsychiatric Inventory-Nursing Home (NPI-NH) questionnaire. The threshold for a reduction in $\mathrm{AB}$ that corresponded to a clinically significant improvement in BPSDs was determined by multiple linear regression.

Results: One hundred forty-seven patients were included (mean age $=84.1 \pm 5.2$ years). Using the ADS, a reduction of 2 points in $A B$ in patients with moderate-intensity BPSDs was associated with a clinically significant improvement in the $\mathrm{F} \times \mathrm{S}$ score of the NPI-NH [6.34, 95\% confidence interval (CI) 4.54-8.14], and a reduction of 3 points was associated with a clinically significant improvement in the occupational disruptiveness score (4.26, 95\% CI 3.11-5.41).

Conclusions/Implications: In older patients with dementia presenting BPSDs, the risk-benefit ratio of anticholinergic drugs is debatable and, where possible, drugs with a lower $A B$ would be preferable. Because BPSDs are a frequent cause of hospitalization, a standardized approach to analysis and reduction of $A B$ is warranted in this population. Depending on the scale used to assess anticholinergic burden ( $A B)$, a small reduction in $A B$ is associated with a decrease in frequency, severity, and disruptiveness of moderate-intensity BPSDs. Drugs with a high AB should be avoided where possible in older patients with dementia, and drugs with a lower $A B$ would be preferable. Heterogeneity between the assessment scales for $A B$ precludes generalization of the impact of a reduction in $A B$ on BPSDs.
\end{abstract}

(c) 2018 AMDA - The Society for Post-Acute and Long-Term Care Medicine.

\footnotetext{
The authors declare no conflicts of interest.

* Address correspondence to Rachid Mahmoudi, MD, PhD, Department of Geriatrics and Internal Medicine, Reims University Hospitals, Maison Blanche Hospital, 45 rue Cognacq Jay, Reims, F-51092, France.

E-mail address: rmahmoudi@chu-reims.fr (R. Mahmoudi).
}

Behavioral and psychological symptoms of dementia (BPSDs) are a group of neuropsychiatric symptoms arising from dementia, and include mood disturbances, depression, agitation, psychosis, sleep disorders, anxiety, apathy, dysphoria, aberrant motor behavior, hallucinations, and delusions. ${ }^{1}$ BPSDs affect $90 \%$ of patients with Alzheimer's disease (AD) and are also common in other types of dementia. $^{2}$ They are a frequent cause of hospitalization, ${ }^{3}$ and are 
associated with increased mortality, ${ }^{4}$ an increased risk of nursing home placement, ${ }^{5}$ caregiver burden, ${ }^{6}$ and more rapid progression of dementia. ${ }^{7}$ A range of assessment tools are available to evaluate BPSDs, including the Neuropsychiatric Inventory-Nursing Home (NPI-NH) tool. ${ }^{8}$ To date, there is no consensus regarding the optimal medical treatment for BPSDs. Nonpharmacologic interventions are preferred, because they are associated with fewer adverse effects than drugs, for a similar effect size. ${ }^{9}$

Certain symptomatic treatments used for BPSDs possess anticholinergic properties, known as the anticholinergic burden (AB).$^{10}$ Anticholinergic drugs encompass several different therapeutic classes (such as certain psychotropic agents, drugs to treat urinary incontinence, painkillers, antihistamine drugs, and some heart failure treatments). Furthermore, although these drugs are known to be potentially inappropriate for older patients, ${ }^{11}$ their use is widespread for the treatment of BPSDs. ${ }^{12}$

The association between $A B$ and BPSDs in older patients with dementia remains unclear. A recent study in an older population of outpatients with subjective cognitive decline or neurocognitive disorders reported that exposure to anticholinergic medications [assessed using the Anticholinergic Cognitive Burden Scale (ACB)] ${ }^{13}$ was associated with higher NPI scores. ${ }^{14}$ However, in this study, after adjustment for potential confounders, the $A B$ was not significantly associated with the NPI-NH score. Furthermore, a relationship has previously been established between the use of drugs with anticholinergic properties and the occurrence of BPSDs, such as hallucinations or delusions. ${ }^{15}$ Among older hospitalized patients, we recently showed that a reduction of the $A B$, as evaluated by the $A C B$, made it possible to reduce the frequency, severity, and disruptiveness of BPSDs. ${ }^{16}$

In this context, the aim of this study was to determine the threshold of reduction in $A B$ that would lead to a clinically significant improvement in the frequency $\times$ severity $(\mathrm{F} \times \mathrm{S})$ score of BPSDs as assessed by the NPI-NH. The secondary objective was to determine the threshold of reduction in $A B$ that would lead to a clinically significant decrease in the occupational disruptiveness (OD) score, as assessed by the NPI-NH.

\section{Methods}

\section{Study Sample}

We included patients aged 65 years or older who were followed for dementia, and hospitalized for BPSDs in a dedicated geriatric care unit specializing in the management of patients with dementia, between July 15, 2015, and October 31, 2017.

\section{Study Design}

This was a single-center, prospective study performed in a dedicated geriatric unit specializing in the management of patients with dementia at the Reims University Hospital, France. How this unit functions has previously been described elsewhere. ${ }^{17}$ Dementia diagnosis was established by senior practitioners (neurologist or geriatrician) according to Diagnostic and Statistical Manual of Mental Disorders, 4 th edition (DSM-IV) criteria. ${ }^{18}$

An initial evaluation of $A B$ was performed at the patient's admission, using the last available prescription detailing the treatment ongoing at the time of hospitalization. A second evaluation of $\mathrm{AB}$ was performed before discharge, based on the discharge prescription. Medications with anticholinergic properties were replaced during the hospital stay, where possible, with other drugs that had lesser or no anticholinergic activity.

BPSDs were evaluated using the NPI-NH by the caregiving team (psychologist, nurses, and/or nurses' aides from the unit; all are trained in the administration of this instrument) during a multidisciplinary meeting. The first score was calculated 2 days after admission, and the second score was calculated 2 days before discharge. Because the physicians in the unit were the prescribers, they did not participate in the evaluation of BPSDs in order not to influence the NPI-NH scores or any potential changes to medication.

\section{Assessment of $A B$ Exposure}

The $A B$ was evaluated for each patient using 3 different instruments, namely, the Anticholinergic Drug Scale (ADS), ${ }^{19}$ the $\mathrm{ACB},{ }^{13}$ and the Anticholinergic Risk Scale (ARS). ${ }^{20}$ The details of the score calculation for each of these scales is provided in Appendix 1 of the Supplementary Data.

\section{BPSD Assessment}

BPSDs were assessed using the Neuropsychiatric Inventory-Nursing Home scale (NPI-NH). The $\mathrm{F} \times \mathrm{S}$ and $\mathrm{OD}$ scores were summed for each patient. The NPI-NH covers 12 domains (delusions, hallucinations, agitation/aggression, depression/ dysphoria, anxiety, elation/euphoria, apathy, disinhibition, irritability/ lability, aberrant motor behavior, sleep and nighttime behavior disorders, and appetite and eating changes). The frequency score of each domain is rated from 1 (less than once a week) to 4 (at least once a day or continuously present). The severity score is rated from 1 (present but not distressing to the patient) to 3 (very stressful and upsetting; typically requires specific management). The OD score measures how disruptive the caregivers find the patient's behavior to their workload, and is rated from 0 (not at all) to 5 (very severely or extremely).

\section{Covariates}

We recorded sociodemographic data (age, sex) and the variables of the standard comprehensive geriatric assessment. The level of independence at baseline was assessed using Katz's Activities of Daily Living (ADL) scale. ${ }^{21}$ Cognitive impairment was assessed using the Mini-Mental State Examination (MMSE). ${ }^{22}$ Mood was assessed using the Mini-Geriatric Depression Scale ${ }^{23}$ if MMSE score was $\geq 15$, or by Cornell scale ${ }^{24}$ if MMSE was $<15$. A modified version of the Charlson Comorbidity Index was used to estimate comorbidity burden. ${ }^{25}$ Nutritional status was assessed using the Mini-Nutritional Assessment. $^{26}$

\section{Clinical Outcomes}

The primary endpoint was a clinically significant decrease in the $\mathrm{F} \times \mathrm{S}$ score of the NPI-NH, defined as a decrease of 4 points or more, as described by Cummings et al. $^{27}$ The secondary endpoint was a clinically significant decrease in the OD score of 3.10 to 3.95 points, reported by Mao et al to be associated with a significant decrease in OD. ${ }^{28}$

\section{Statistical Analysis}

Quantitative data are described as mean \pm standard deviation and categorical variables as number (percentage). The $\mathrm{F} \times \mathrm{S}$ and $\mathrm{OD}$ scores of the NPI-NH (at baseline) were categorized into tertiles. In both univariate and multivariate analysis, only patients whose $A B$ was greater than 0 at inclusion were included. Simple linear regression to model the decrease in $\mathrm{F} \times \mathrm{S}$ score of the NPI-NH was performed for each candidate variable. Clinical variables of interest (age, sex, level of ADL, type of dementia, stage of dementia, likelihood of depression, comorbidity burden, and nutritional status) were included in the 
multivariate model using an ascending stepwise approach, with a threshold for exit from the model set at 0.05 . One model was constructed for each scale. The relevant $A B$ scale score was forced in the corresponding model. We tested interactions between the reduction in $\mathrm{AB}$, initial $\mathrm{AB}$, and initial level of BPSDs. The relationships between continuous variables and NPI-NH score $(\mathrm{F} \times \mathrm{S}$ or $\mathrm{OD})$ were analyzed to ensure a linear relationship, and if it was not found to be linear, a quadratic term was added. The same tests were performed to model the OD score of the NPI-NH. The alpha risk was set at 0.05 . The goodness of fit of the multiple linear regression models was estimated using the adjusted $R$-squared. All analyses were performed using $\mathrm{R}$ software [R Core Team (2014)].

\section{Ethical Considerations}

This study was approved by the Institutional Review Board of the Reims University Hospital, France, under the number 2016-16.

\section{Results}

\section{Characteristics of the Study Population}

A total of 147 patients were included (average age was $84.1 \pm$ 5.2 years). Eighty patients (54.4\%) had AD, 42 (28.6\%) had mixed dementia (AD and vascular), 20 (13.6\%) had vascular dementia, and 5 had Lewy body dementia. The characteristics of the study population are displayed in Table 1.

Overall, 80 (54.4\%) of the patients had a reduction in $A B$ as assessed by the ACB, 66 (44.9\%) when assessed by the ADS, and 77 (52.4\%) when assessed by the ARS.

\section{Decrease in F $\times$ S Score of the NPI-NH, According to the Change in $A B$}

According to the ADS, there was a significant overall association between the reduction in $A B$ and the decrease in the $F \times S$ score of the NPI-NH (Figure $1 \mathrm{~A}$ and Table 2). A reduction of 2 points in $A B$ was associated with a clinically significant decrease in the $\mathrm{F} \times \mathrm{S}$ score [6.34, 95\% confidence interval (CI) 4.54-8.14] in subjects with moderately intense BPSDs (initial $\mathrm{F} \times \mathrm{S}$ score between 10 and 20) (Figure $1 \mathrm{~B}$ and Table 2). No other clinical variable was significantly associated with a decrease in $\mathrm{F} \times \mathrm{S}$ score.

Using the ACB and ARS scales, there was no significant relationship between a reduction in $\mathrm{AB}$ and a decrease in $\mathrm{F} \times \mathrm{S}$ score of the NPI-NH (Table 2 and Appendices 2 and 3).

\section{Decrease in the OD Score of the NPI-NH, According to the Change in} $A B$

Using the ADS, there was a significant overall association between the reduction in $\mathrm{AB}$ and a clinically significant decrease in the OD score of the NPI-NH (Figure $1 \mathrm{C}$ and Table 2). A reduction of 3 points in $\mathrm{AB}$ as assessed by the ADS led to a clinically significant decrease of more than 3 points in the OD score $(4.26,95 \% \mathrm{CI} 3.11-5.41)$ in patients with moderate-intensity BPSDs (initial OD score between 5 and 10) (Figure 1D and Table 2). No other clinical variable was related to a significant decrease in the OD score.

According to the ACB and ARS scores, there was no significant relation between a reduction in $\mathrm{AB}$ and a decrease in the OD score (Table 2, Appendices 2 and 3).

The decrease in BPSDs at each cut-off point for the reduction in AB (in 1-point increments) according to the 3 assessment scales is detailed in Table 2.
Table 1

Characteristics of the Study Population $(\mathrm{N}=147)$

\begin{tabular}{|c|c|}
\hline Variables & \\
\hline Age, mean \pm SD & $84.1 \pm 5.2$ \\
\hline Age $65-75$ years, $n(\%)$ & $4(2.7)$ \\
\hline Age $75-85$ years, $n(\%)$ & $53(36.1)$ \\
\hline Age 85 years or more, $\mathrm{n}(\%)$ & $90(61.2)$ \\
\hline Female sex, $\mathrm{n} \%$ & $95(64.6)$ \\
\hline $\mathrm{ADL} \geq 3, \mathrm{n}(\%)$ & $108(73.5)$ \\
\hline MMSE, mean \pm SD & $13.3 \pm 5.3$ \\
\hline \multicolumn{2}{|l|}{ Type of dementia, $\mathrm{n}(\%)$} \\
\hline $\mathrm{AD}$ & $80(54.4)$ \\
\hline Vascular & $20(13.6)$ \\
\hline LBD & $5(3.4)$ \\
\hline Mixed & $42(28.6)$ \\
\hline $\begin{array}{l}\text { At risk of depression (Mini-GDS score } \geq 1 \text { or } \\
\text { Cornell score } \geq 10), \mathrm{n}(\%)\end{array}$ & $84(57.9)$ \\
\hline \multicolumn{2}{|l|}{ Charlson Comorbidity Index, n (\%) } \\
\hline Mild comorbidity (1-2) & $45(30.6)$ \\
\hline Moderate to severe comorbidity $(\geq 3)$ & $102(69.4)$ \\
\hline At risk of undernutrition or malnourished, $n(\%)$ & $104(70.4)$ \\
\hline Length of stay, $\mathrm{d}$, mean $\pm \mathrm{SD}$ & $11.2 \pm 3.1$ \\
\hline \multicolumn{2}{|l|}{ BPSDs prevalence according to NPI-NH, $\mathrm{n}(\%)$} \\
\hline Delusions & $38(25.9)$ \\
\hline Hallucinations & $24(16.3)$ \\
\hline Agitation/aggression & $57(39.2)$ \\
\hline Depression/dysphoria & $50(34.0)$ \\
\hline Anxiety & $69(34.7)$ \\
\hline Elation/euphoria & 0 \\
\hline Apathy/indifference & $47(32.0)$ \\
\hline Disinhibition & $5(3.4)$ \\
\hline Irritability/lability & $57(38.7 .4)$ \\
\hline Aberrant motor behavior & $57(38.7)$ \\
\hline Sleep and nighttime behavior disorders & $52(35.4)$ \\
\hline $\mathrm{NPI}-\mathrm{NH} \mathrm{F} \times \mathrm{S}$ score at baseline, mean $\pm \mathrm{SD}$ & $13.5 \pm 11.6$ \\
\hline NPI-NH OD score at baseline, mean \pm SD & $7.3 \pm 4.7$ \\
\hline $\mathrm{AB}$ at baseline $>0$ assessed by ADS scale, $\mathrm{n}(\%)$ & $123(83.7)$ \\
\hline ADS score at baseline, mean \pm SD & $2.2 \pm 1.7$ \\
\hline $\mathrm{AB}$ at baseline $>0$ assessed by $\mathrm{ACB}$ scale, $\mathrm{n}(\%)$ & $118(80.3)$ \\
\hline ACB score at baseline, mean \pm SD & $2.2 \pm 1.7$ \\
\hline $\mathrm{AB}$ at baseline $>0$ assessed by ARS scale, $\mathrm{n}(\%)$ & $126(85.7)$ \\
\hline ARS score at baseline, mean \pm SD & $2.3 \pm 1.8$ \\
\hline
\end{tabular}

LBD, Lewy body dementia; Mini-GDS, Mini Geriatric Depression Scale; SD, standard deviation.

Missing data: nutritional status, $\mathrm{n}=8$; probability of depression, $\mathrm{n}=2$.

\section{Discussion}

This study shows that based on ADS scores, a small reduction in $\mathrm{AB}$ is associated with a clinically significant decrease in moderateintensity BPSDs in older subjects with dementia, independently of the other variables investigated. As reported by Lövheim et $\mathrm{al}^{29}{ }^{29}$ our results may be related to the higher prevalence of BPSDs in older subjects at the middle stages of dementia, regardless of the type of dementia considered. The mean MMSE score in our population was $13.3 \pm 5.3$.

According to our findings, regardless of the scale used to assess $A B$, there was no significant effect of a reduction in $A B$ among patients presenting mild BPSDs. Indeed, since the starting score for BPSDs was low, it is difficult to achieve a clinically significant decrease of 4 points, due to a sort of "floor" effect. Indeed, in our population, $96.6 \%$ of patients had $A D$, vascular dementia, or mixed dementia (vascular and AD). According to Tanaka et $\mathrm{al}^{30}{ }^{30}$ the severity of BPSDs as evaluated by the NPI progresses with dementia stage in subjects with AD. Furthermore, a cholinergic deficit may also be involved in vascular dementia. In this regard, Tohgi et al showed that the level of acetylcholine in the cerebrospinal fluid of subjects with vascular disease was $49 \%$ lower than that of controls. ${ }^{31}$ Therefore, in our study population, with mild BPSDs, it is possible that the cholinergic deficit was not sufficiently marked for a reduction of $A B$ to lead to a decrease in BPSDs. 
A

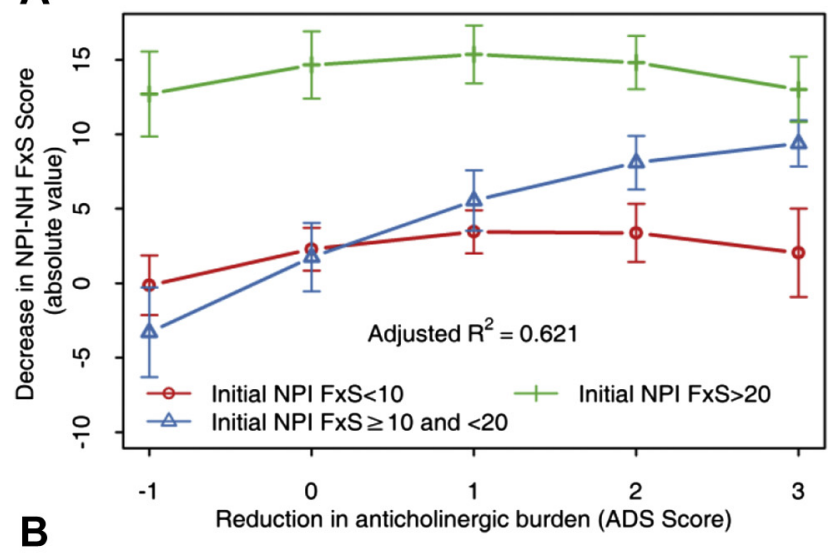

B

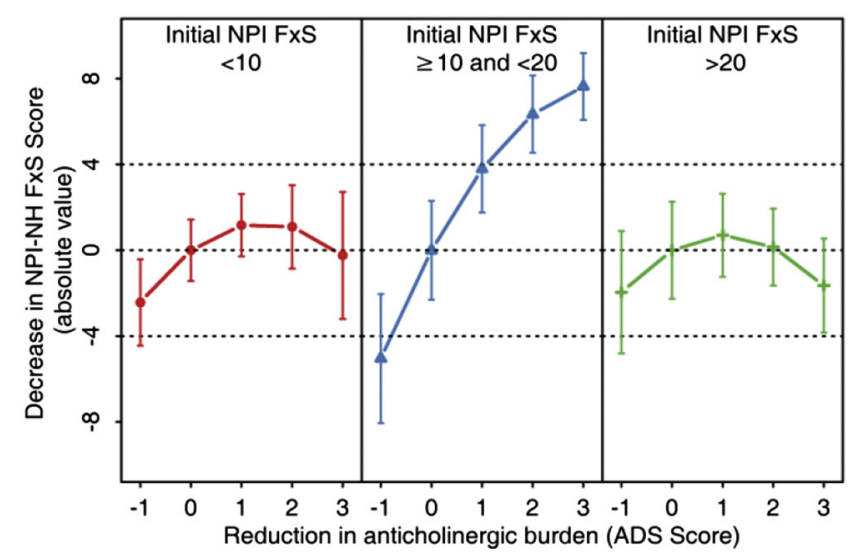

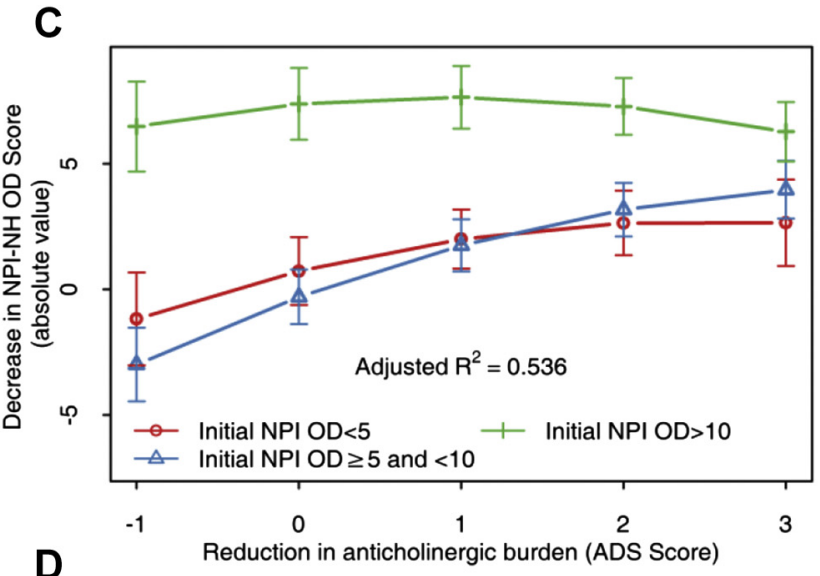

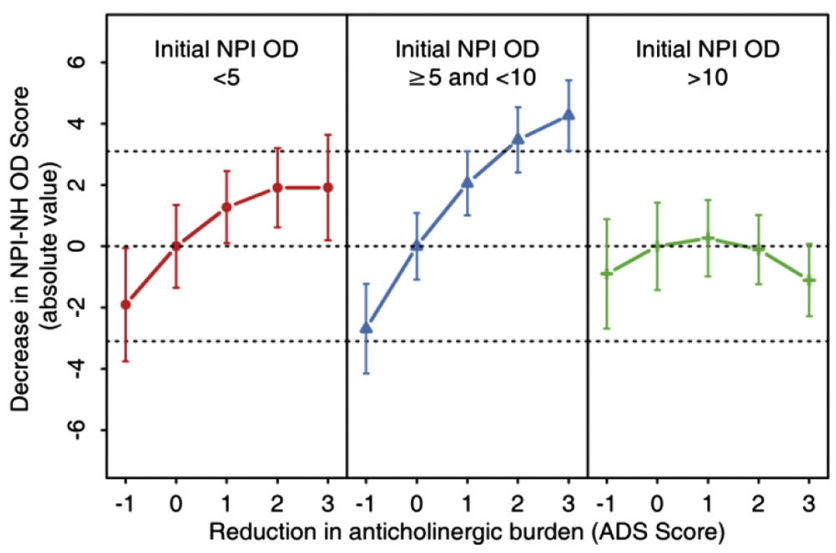

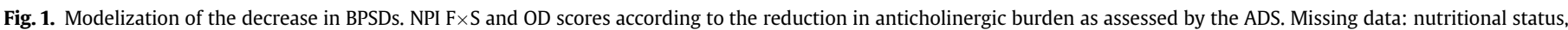

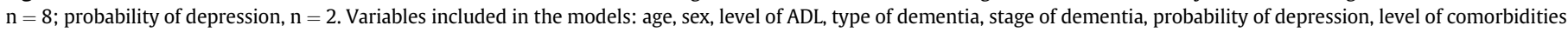

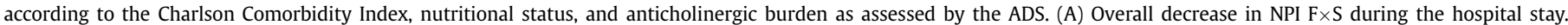
(B) Decrease in NPI F $\times$ S according to initial BPSDs. (C) Overall decrease in NPI OD during the hospital stay. (D) Decrease in NPI OD according to initial BPSDs.

Conversely, whatever the reduction in $A B$, we observed a substantial decrease in BPSDs in patients who had severe BPSDs at admission, albeit without observing a "dose-response" relationship in the reduction. Accordingly, the reduction in $\mathrm{AB}$ alone could not induce a decrease in BPSDs, as there was evidence of a "ceiling" effect. Indeed, multiple etiologic factors are implicated in the emergence and persistence of BPSDs, such as the type of dementia, ${ }^{32}$ the severity of cognitive impairment, ${ }^{33}$ and neurotransmitter deficits, ${ }^{34}$ as well as

Table 2

Decrease in BPSDs According to the Reduction (in 1-Point Increments) in AB as Assessed by the ADS, ACB, and ARS

\begin{tabular}{|c|c|c|c|c|c|c|}
\hline & \multicolumn{3}{|c|}{ Decrease in $\mathrm{F} \times \mathrm{S}$ Score, Points $(95 \% \mathrm{CI}$ ) } & \multicolumn{3}{|c|}{ Decrease in OD Score, Points (95\% CI) } \\
\hline & Initial Score $<10$ & $\geq 10$ Initial Score $<20$ & Initial Score $\geq 20$ & Initial Score $<5$ & $\geq 5$ Initial Score $<10$ & Initial Score $\geq 10$ \\
\hline \multicolumn{7}{|l|}{ ADS } \\
\hline 1 & $1.17(-0.28,2.62)$ & $3.79(1.76,5.83)$ & $0.70(-1.229,2.63)$ & $1.27(0.10,2.44)$ & $2.05(1.01,3.09)$ & $0.26(-0.98,1.51)$ \\
\hline 2 & $1.09(-0.86,3.04)$ & $6.34(4.54,8.14)^{*}$ & $0.16(-1.64,1.95)$ & $1.91(0.62,3.2)$ & $3.47(2.41,4.54)$ & $-0.10(-1.23,1.02)$ \\
\hline 3 & $-0.24(-3.20,2.72)$ & $7.63(6.08,9.19)^{*}$ & $-1.64(-3.83,0.54)$ & $1.92(0.20,3.63)$ & $4.26(3.11,5.41)^{\dagger}$ & $-1.10(-2.28,0.07)$ \\
\hline \multicolumn{7}{|l|}{$\mathrm{ACB}$} \\
\hline 1 & $1.37(-0.04,2.79)$ & $3.00(1.32,4.68)$ & $1.13(-0.64,2.91)$ & $1.29(0.20,2.37)$ & $2.14(1.19,3.08)$ & $0.56(-0.48,1.60)$ \\
\hline 2 & $1.37(-0.35,3.09)$ & $4.63(3.22,6.05)$ & $0.89(-0.89,2.67)$ & $1.81(0.64,2.98)$ & $3.51(2.19,4.41)$ & $0.35(-0.61,1.32)$ \\
\hline 3 & $-0.01(-2.39,2.37)$ & $4.89(3.53,6.24)$ & $-0.72(-3.27,1.81)$ & $1.57(-0.02,3.16)$ & $4.12(3.07,5.17)$ & $-0.62(-1.76,0.52)$ \\
\hline \multicolumn{7}{|r|}{ 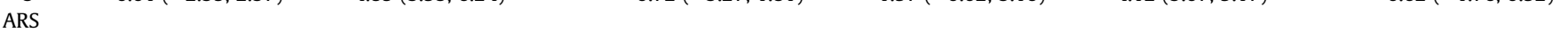 } \\
\hline 1 & $1.38(0.21,2.56)$ & $2.33(1.29,3.37)$ & $0.69(-0.56,1.93)$ & $1.38(0.21,2.56)$ & $2.33(1.29,3.37)$ & $0.69(-0.56,1.93)$ \\
\hline 2 & $1.51(0.22,2.80)$ & $3.41(2.35,4.47)$ & $0.12(-1.00,1.25)$ & $1.51(0.22,2.80)$ & $3.41(2.347,4.47)$ & $0.12(-1.003,1.25)$ \\
\hline 3 & $0.39(-1.325,2.11)$ & $3.24(2.09,4.38)$ & $-1.69(-2.87,-0.51)$ & $0.39(-1.325,2.11)$ & $3.24(2.09,4.38)$ & $-1.69(-2.87,-0.51)$ \\
\hline
\end{tabular}

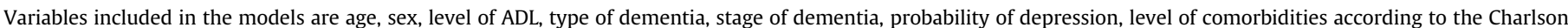
Comorbidity Index, nutritional status, and anticholinergic burden as assessed by the ADS, ACB, and ARS.

Linear regression model: decrease in $\mathrm{F} \times \mathrm{S}$ score according to $\mathrm{ACB}$ : adjusted $R$-squared $=0.62 ; \mathrm{F}$-statistic $=33.41$ on 6 ; $\mathrm{df}=114 ; P<.01$

Linear regression model: decrease in $\mathrm{F} \times \mathrm{S}$ score according to $\mathrm{AD}$ : adjusted $R$-squared $=0.60 ; \mathrm{F}$-statistic $=29.78$ on $6 ; \mathrm{df}=109 ; P<.01$. Linear regression model: decrease in $\mathrm{F} \times \mathrm{S}$ score according to ARS: adjusted $R$-squared $=0.69 ; \mathrm{F}$-statistic $=43.55$ on $6 ; \mathrm{df}=117 ; P<.01$. Linear regression model: decrease in OD score according to ACB: adjusted $R$-squared $=0.58 ; \mathrm{F}$-statistic $=29.39$ on $6 ; \mathrm{df}=114 ; P<.01$. Linear regression model: decrease in OD score according to ADS: adjusted $R$-squared $=0.53 ; \mathrm{F}$-statistic $=21.00$ on $6 ; \mathrm{df}=109 ; P<.01$. Linear regression model: decrease in OD score according to ARS: adjusted $R$-squared $=0.67 ;$ F-statistic $=39.73$ on $6 ; \mathrm{df}=117 ; P<.01$.

*Clinically significant $(>4$ for $\mathrm{F} \times \mathrm{S}$ score) and statistically significant $(P<.05)$.

${ }^{\dagger}$ Clinically significant $(>3.1$ for $\mathrm{OD})$ and statistically significant $(P<.05)$. 
vascular $^{35}$ and environmental determinants. ${ }^{36}$ At the severe level of BPSDs, it is possible that these etiologic factors may be preponderant and have a greater impact than the cholinergic deficit in the emergence and persistence of BPSDs.

Furthermore, in our study, beyond a certain threshold, further reduction in $A B$ failed to lead to further decrease in BPSDs. In our study population, the decrease in BPSDs could be partially due to the multidisciplinary management in line with expert recommendations within our specialized geriatric unit. ${ }^{37}$

We observed differences in our findings depending on the score used to assess $\mathrm{AB}$. Accordingly, the reduction in $\mathrm{AB}$ as assessed by the ADS was associated with a significant decrease in BPSDs, whereas no such significant relation was observed when the ACB or ARS were used. This is likely to be the consequence of the heterogeneity between these different tools to evaluate $A B$, and in their average level of agreement with each other. ${ }^{38}$ A recent study showed substantial disparities between 9 available instruments for evaluating $A B$, and the clinical consequences this could have in older patients with dementia. ${ }^{39}$ As discussed by Turro-Garigra et al in their study, there were substantial differences in some outcomes (such as hypertension, stage of dementia, depression, diabetes mellitus) across the 9 anticholinergic risk scales, including the ADS, ACB, and ARS. ${ }^{39}$ Furthermore, the same drug may be evaluated differently by different scales. For example, the $A B$ of risperidone is scored 1 by the $A C B$ and $A R S$, and 0 on the ADS. In this context, it is difficult to ascertain the true magnitude of the effect of a reduction in $A B$ on BPSDs. The use of these instruments in clinical practice or for research purposes requires the user to be aware of their respective limitations. Among the 3 scales used here, the ADS includes the largest number of cholinergic antagonists (117 for the ADS, 94 in the ACB and 50 for the ARS). The ADS is the only tool among the 3 to take into account an estimation of $\mathrm{AB}$ based on both biological parameters (namely the association with serum anticholinergic activity $)^{40}$ and clinical parameters, that is, the adverse effects of cholinergic antagonists. Conversely, the design of the ACB is based solely on expert opinion formulated from a review of the literature. ${ }^{13} \mathrm{~A}$ limitation to the use of the ARS in older adults with dementia is that the cognitive status of the population used to develop the ARS was not specified. ${ }^{20}$ Finally, the drugs on all 3 scales include some medications that are not commercially available in France, such as molindone (ADS and ACB), asenapine (ACB), and darifenacin and meclizine (all 3 scales). In fact, as of this writing, there is no single, standardized tool that can unequivocally assess $\mathrm{AB}^{41}$ To meet this need, Duran et al listed 100 medications frequently prescribed in older polymedicated patients, namely, drugs with clinically relevant anticholinergic properties (47 high potency and 53 low potency), and for which there was agreement across the different scales. ${ }^{42}$ However, despite their differences, scales to evaluate $A B$ remain a simple and noninvasive way to estimate the exposure of older patients with dementia to anticholinergic load.

In this context, cholinergic antagonists could represent an avoidable iatrogenic contributing factor to BPSDs, facilitating the onset and persistence of BPSDs, perhaps by compounding a pre-existing cholinergic deficit. Given that there is currently no standardized symptomatic treatment strategy for BPSDs, it is clearly preferable to avoid the prescription of medications that may promote this condition. Our results show one interesting concrete finding: discontinuation of certain medications with a low $\mathrm{AB}$ (eg, codeine, colchicine, cetirizine) contributes to a decrease of moderate-intensity BPSDs in older patients. Indeed, discontinuation or substitution of these medications (whose $A B$ is assessed differently depending on the instrument used) could be easier in routine practice than the interruption of certain other drugs with a higher $\mathrm{AB}$ (eg, amitriptyline, clomipramine, paroxetine, or hydroxyzine). A recent randomized trial tested an intervention aimed at reducing $\mathrm{AB}$ in older patients, especially those with cognitive impairment. ${ }^{43}$
A certain number of covariables were taken into account in our statistical analysis, as we considered them to be of relevance to this study. Advanced age has previously been shown to be associated with the severity of BPSDs, ${ }^{44}$ although sex was also taken into account as there is evidence of differences in BPSD prevalence between men and women. ${ }^{45}$ The level of ADL was also taken into consideration, because in late-stage dementia mobility may be more severely impaired, which can in turn reduce the prevalence of certain BPSDs, such as agitation or wandering. The risk of depression was also considered relevant because psychological signs of depression in older adults can present as agitation or psychosis. ${ }^{46}$ Furthermore, comorbidities were also controlled for, because the presence of multiple comorbidities is reportedly related to the risk of adverse drug reactions, ${ }^{47}$ which, in populations similar to ours, most frequently present in the form of neuropsychiatric disorders. ${ }^{48}$ Finally, risk of malnutrition was also considered relevant to our analysis, because it has been shown that there is an increased risk of malnutrition, as evaluated by the Mini-Nutritional Assessment, in older patients with BPSDs ${ }^{49}$; moreover, BPSDs may lead to weight loss, which in turn promotes malnutrition. ${ }^{50}$

Certain limitations of our study deserve to be underlined. First, this was a single-center study and included a limited number of patients. Second, the estimation of $A B$ was based on available assessment scales and not on the biological measurement of serum anticholinergic activity or in the spinal fluid, which yields more accurate measures. Third, our findings cannot be generalized to all older patients with dementia, since the participants in this study were recruited in a dedicated geriatric care unit specializing in the management of patients with dementia. Finally, the scales used to measure $A B$ do not take into account the dose effect or the duration of exposure to treatment. Strong points of our study include the fact that, to our knowledge, this is the first study to investigate thresholds for a reduction in $A B$ that would have a clinically significant impact on BPSDs. In addition, most participants in the study presented BPSDs that were disruptive and had a negative effect on the patients themselves as well as on their caregivers. This population therefore has the potential to gain substantial benefit from any reduction in $A B$.

\section{Conclusions/Relevance}

In older patients with dementia hospitalized in a dedicated geriatric care unit, a small reduction in $\mathrm{AB}$ as assessed by the ADS is associated with a clinically significant decrease in the $\mathrm{F} \times \mathrm{S}$ and $\mathrm{OD}$ scores of moderate-intensity BPSDs as evaluated by the NPI-NH. Because BPSDs are a frequent cause of hospitalization, a standardized approach to analysis and reduction of $A B$ is warranted in this population. Furthermore, it would be useful to attempt, whenever possible, to prevent BPSDs by substituting cholinergic antagonists with other drugs, or by discontinuing them when already prescribed. Larger-scale studies in other populations of older patients with BPSDs (eg, outpatients, nursing home residents) are warranted to confirm these findings.

\section{Acknowledgments}

The authors thank the caregiving team of the dedicated geriatric care unit specializing in the management of patients with dementia for their help in bringing this project to fruition. The authors thank Fiona Ecarnot (EA3920, University Hospital Besancon, France) for translation and editorial assistance.

\section{References}

1. Lyketsos CG, Steinberg M, Tschanz JT, et al. Mental and behavioral disturbances in dementia: Findings from the Cache County Study on Memory in Aging. Am J Psychiatry 2000;157:708-714. 
2. Steinberg M, Shao H, Zandi P, et al. Point and 5-year period prevalence of neuropsychiatric symptoms in dementia: The Cache County Study. Int J Geriatr Psychiatry 2008;23:170-177.

3. Nourhashemi F, Andrieu S, Sastres N, et al. Descriptive analysis of emergency hospital admissions of patients with Alzheimer disease. Alzheimer Dis Assoc Disord 2001;15:21-25.

4. Lattanzio F, Corica F, Schepisi R, et al. Anticholinergic burden and 1-year mortality among older patients discharged from acute care hospital. Geriatr Gerontol Int 2018;18:705-713.

5. Chan DC, Kasper JD, Black BS, et al. Presence of behavioral and psychological symptoms predicts nursing home placement in community-dwelling elders with cognitive impairment in univariate but not multivariate analysis. J Gerontol A Biol Sci Med Sci 2003;58:548-554.

6. van der Lee J, Bakker TJ, Duivenvoorden HJ, et al. Multivariate models of subjective caregiver burden in dementia: A systematic review. Ageing Res Rev 2014; 15:76-93.

7. Peters ME, Rosenberg PB, Steinberg M, et al. Neuropsychiatric symptoms as risk factors for progression from CIND to dementia: The Cache County Study. Am J Geriatr Psychiatry 2013;21:1116-1124.

8. Wood S, Cummings JL, Hsu MA, et al. The use of the neuropsychiatric inventory in nursing home residents. Characterization and measurement. Am J Geriatr Psychiatry 2000;8:75-83.

9. Dyer SM, Harrison SL, Laver K, et al. An overview of systematic reviews of pharmacological and non-pharmacological interventions for the treatment of behavioral and psychological symptoms of dementia. Int Psychogeriatr 2018; 30:295-309.

10. Fox C, Livingston G, Maidment ID, et al. The impact of anticholinergic burden in Alzheimer's dementia-The LASER-AD study. Age Ageing 2011;40:730-735.

11. American Geriatrics Society Beers Criteria Update Expert Panel. American Geriatrics Society 2015 updated Beers criteria for potentially inappropriate medication use in older adults. J Am Geriatr Soc 2015;63:2227-2246.

12. Jessen F, Kaduszkiewicz H, Daerr M, et al. Anticholinergic drug use and risk for dementia: Target for dementia prevention. Eur Arch Psychiatry Clin Neurosci 2010;260(suppl 2):S111-S115

13. Boustani M, Campbell N, Munger S, et al. Impact of anticholinergics on the aging brain: A review and practical application. Aging Health 2008;4: $411-420$.

14. Dauphinot V, Mouchoux C, Veillard S, et al. Anticholinergic drugs and functional, cognitive impairment and behavioral disturbances in patients from a memory clinic with subjective cognitive decline or neurocognitive disorders. Alzheimers Res Ther 2017;9:58.

15. Cancelli I, Valentinis L, Merlino G, et al. Drugs with anticholinergic properties as a risk factor for psychosis in patients affected by Alzheimer's disease. Clin Pharmacol Ther 2008;84:63-68.

16. Jaidi Y, Nonnonhou V, Kanagaratnam L, et al. Reduction of the anticholinergic burden makes it possible to decrease behavioral and psychological symptoms of dementia. Am J Geriatr Psychiatry 2018;26:280-288.

17. Mahmoudi R, Dramé M, Morrone I, et al. [A short-term stay ward for patients with Alzheimer's disease and associated disorders: The experience of the Reims Teaching Hospital]. NPG Neurol-Psychiatr-Gériatrie 2012;12:230-236.

18. American Psychiatry Association. In: DSM-IV, Diagnosis and Statistical Manual of Mental Disorders. 4th ed. Washington, DC: American Psychiatry Association; 1994.

19. Carnahan RM, Lund BC, Perry PJ, et al. The Anticholinergic Drug Scale as a measure of drug-related anticholinergic burden: Associations with serum anticholinergic activity. J Clin Pharmacol 2006;46:1481-1486.

20. Rudolph JL, Salow MJ, Angelini MC, et al. The anticholinergic risk scale and anticholinergic adverse effects in older persons. Arch Intern Med 2008;168: 508-513.

21. Katz S. Assessing self-maintenance: Activities of daily living, mobility, and instrumental activities of daily living. J Am Geriatr Soc 1983;31:721-727.

22. Folstein MF, Folstein SE, McHugh PR. "Mini-mental state." A practical method for grading the cognitive state of patients for the clinician. J Psychiatr Res 1975; 12:189-198.

23. Yesavage JA, Brink TL, Rose TL, et al. Development and validation of a geriatric depression screening scale: A preliminary report. J Psychiatr Res 1982;17: $37-49$.

24. Alexopoulos GS, Abrams RC, Young RC, Shamoian CA. Cornell scale for depression in dementia. Biol Psychiatry 1988;23:271-284.

25. Sundararajan V, Henderson T, Perry C, et al. New ICD-10 version of the Charlson comorbidity index predicted in-hospital mortality. J Clin Epidemiol 2004;57: 1288-1294.
26. Guigoz Y, Vellas B, Garry PJ. Assessing the nutritional status of the elderly: The Mini Nutritional Assessment as part of the geriatric evaluation. Nutr Rev 1996; 54(1, pt 2):S59-S65.

27. Cummings JL. Changes in neuropsychiatric symptoms as outcome measures in clinical trials with cholinergic therapies for Alzheimer disease. Alzheimer Dis Assoc Disord 1997;11(suppl 4):S1-S9.

28. Mao HF, Kuo CA, Huang WN, et al. Values of the minimal clinically important difference for the Neuropsychiatric Inventory Questionnaire in individuals with dementia. J Am Geriatr Soc 2015;63:1448-1452.

29. Lovheim H, Sandman PO, Karlsson S, et al. Behavioral and psychological symptoms of dementia in relation to level of cognitive impairment. Int Psychogeriatr 2008;20:777-789.

30. Tanaka H, Hashimoto M, Fukuhara R, et al. Relationship between dementia severity and behavioural and psychological symptoms in early-onset Alzheimer's disease. Psychogeriatrics 2015;15:242-247.

31. Tohgi H, Abe T, Kimura M, et al. Cerebrospinal fluid acetylcholine and choline in vascular dementia of Binswanger and multiple small infarct types as compared with Alzheimer-type dementia. J Neural Trans 1996;103:1211-1220.

32. McKeith I, Cummings J. Behavioural changes and psychological symptoms in dementia disorders. Lancet Neurol 2005;4:735-742.

33. Srikanth S, Nagaraja AV, Ratnavalli E. Neuropsychiatric symptoms in dementia-frequency, relationship to dementia severity and comparison in Alzheimer's disease, vascular dementia and frontotemporal dementia. J Neurol Sci 2005;236:43-48.

34. Lanari A, Amenta F, Silvestrelli G, et al. Neurotransmitter deficits in behavioural and psychological symptoms of Alzheimer's disease. Mech Ageing Dev 2006; 127:158-165.

35. Steinberg M, Hess K, Corcoran C, et al. Vascular risk factors and neuropsychiatric symptoms in Alzheimer's disease: The Cache County Study. Int J Geriatr Psychiatry 2014;29:153-159.

36. Feast A, Orrell M, Russell I, et al. The contribution of caregiver psychosocial factors to distress associated with behavioural and psychological symptoms in dementia. Int J Geriatr Psychiatry 2017;32:76-85.

37. Kales HC, Gitlin LN, Lyketsos CG, et al. Management of neuropsychiatric symptoms of dementia in clinical settings: Recommendations from a multidisciplinary expert panel. J Am Geriatr Soc 2014;62:762-769.

38. Naples JG, Marcum ZA, Perera S, et al. Concordance between anticholinergic burden scales. J Am Geriatr Soc 2015;63:2120-2124.

39. Turro-Garriga O, Calvo-Perxas L, Vilalta-Franch J, et al. Measuring anticholinergic exposure in patients with dementia: A comparative study of nine anticholinergic risk scales. Int J Geriatr Psychiatry 2018;33:710-717.

40. Carnahan RM, Lund BC, Perry PJ, et al. The relationship of an anticholinergic rating scale with serum anticholinergic activity in elderly nursing home residents. Psychopharmacol Bull 2002;36:14-19.

41. Salahudeen MS, Duffull SB, Nishtala PS. Anticholinergic burden quantified by anticholinergic risk scales and adverse outcomes in older people: A systematic review. BMC Geriatr 2015;15:31.

42. Duran CE, Azermai M, Vander Stichele RH. Systematic review of anticholinergic risk scales in older adults. Eur J Clin Pharmacol 2013;69:1485-1496.

43. Moga DC, Abner EL, Rigsby DN, et al. Optimizing medication appropriateness in older adults: A randomized clinical interventional trial to decrease anticholinergic burden. Alzheimers Res Ther 2017;9:36.

44. Vaingankar JA, Chong SA, Abdin E, et al. Behavioral and psychological symptoms of dementia: Prevalence, symptom groups and their correlates in community-based older adults with dementia in Singapore. Int Psychogeriatr 2017;29:1363-1376.

45. Lovheim H, Sandman PO, Karlsson S, et al. Sex differences in the prevalence of behavioral and psychological symptoms of dementia. Int Psychogeriatr 2009; 21:469-475.

46. Blazer DG. Depression in late life: Review and commentary. J Gerontol A Biol Sci Med Sci 2003;58:249-265.

47. Onder G, Petrovic M, Tangiisuran B, et al. Development and validation of a score to assess risk of adverse drug reactions among in-hospital patients 65 years or older: The GerontoNet ADR risk score. Arch Intern Med 2010;170:1142-1148.

48. Kanagaratnam L, Mahmoudi R, Novella JL, et al. Adverse drug reactions in elderly subjects hospitalized in a specialized dementia management unit. Drugs Aging 2014;31:769-776.

49. D'Onofrio G, Panza F, Sancarlo D, et al. Delusions in patients with Alzheimer's disease: A multidimensional approach. J Alzheimers Dis 2016;51:427-437.

50. Guérin O, Andrieu S, Schneider SM, et al. Characteristics of Alzheimer's disease patients with a rapid weight loss during a six-year follow-up. Clin Nutr 2009; 28:141-146. 


\section{Appendix 1}

The Anticholinergic Drug Scale (ADS) ${ }^{\mathrm{E} 1}$ was developed based on measures of serum anticholinergic activity and validated in a population of elderly long-term care facility residents. It covers a total of 117 drugs, which are rated from 0 to 3 (with $0=$ no known anticholinergic activity; 1 = potentially anticholinergic as evidenced by receptor-binding studies; 2 = anticholinergic adverse events sometimes noted, usually at excessive doses; and $3=$ marked anticholinergic activity).

The Anticholinergic Cognitive Burden scale $(A C B)^{\mathrm{E} 2}$ was designed to identify the severity of adverse impact of anticholinergic drugs on cognitive function. The ACB lists a total of 88 drugs, rated from 0 to 3 ( 0 corresponding to no anticholinergic effects, $1=$ drugs with possible but not clinically relevant anticholinergic effects; 2 = drugs with established and clinically relevant moderate to severe cognitive effects not associated with development of delirium; 3 = drugs with established and clinically relevant moderate to severe cognitive effects and associated with development of delirium).

The Anticholinergic Risk Scale (ARS) ${ }^{\mathrm{E} 3}$ was developed based on a review of the literature and expert opinion concerning the 500 most prescribed medications within the Veterans Affairs Boston Healthcare System (excluding topical ophthalmic, otologic, and inhaled medication preparations). It lists a total of 49 drugs, whose anticholinergic potential is ranked on a scale from 0 to $3(0=$ limited or non, $1=$ moderate, 2 = strong, and $3=$ very strong).

\section{References}

E1. Carnahan RM, Lund BC, Perry PJ, et al. The Anticholinergic Drug Scale as a measure of drug-related anticholinergic burden: Associations with serum anticholinergic activity. J Clin Pharmacol 2006;46:1481-1486.

E2. Boustani M, Campbell N, Munger S, et al. Impact of anticholinergics on the aging brain: A review and practical application. Aging Health 2008;4: 411-420.

E3. Rudolph JL, Salow MJ, Angelini MC, et al. The anticholinergic risk scale and anticholinergic adverse effects in older persons. Arch Intern Med 2008;168:508-513. 
A
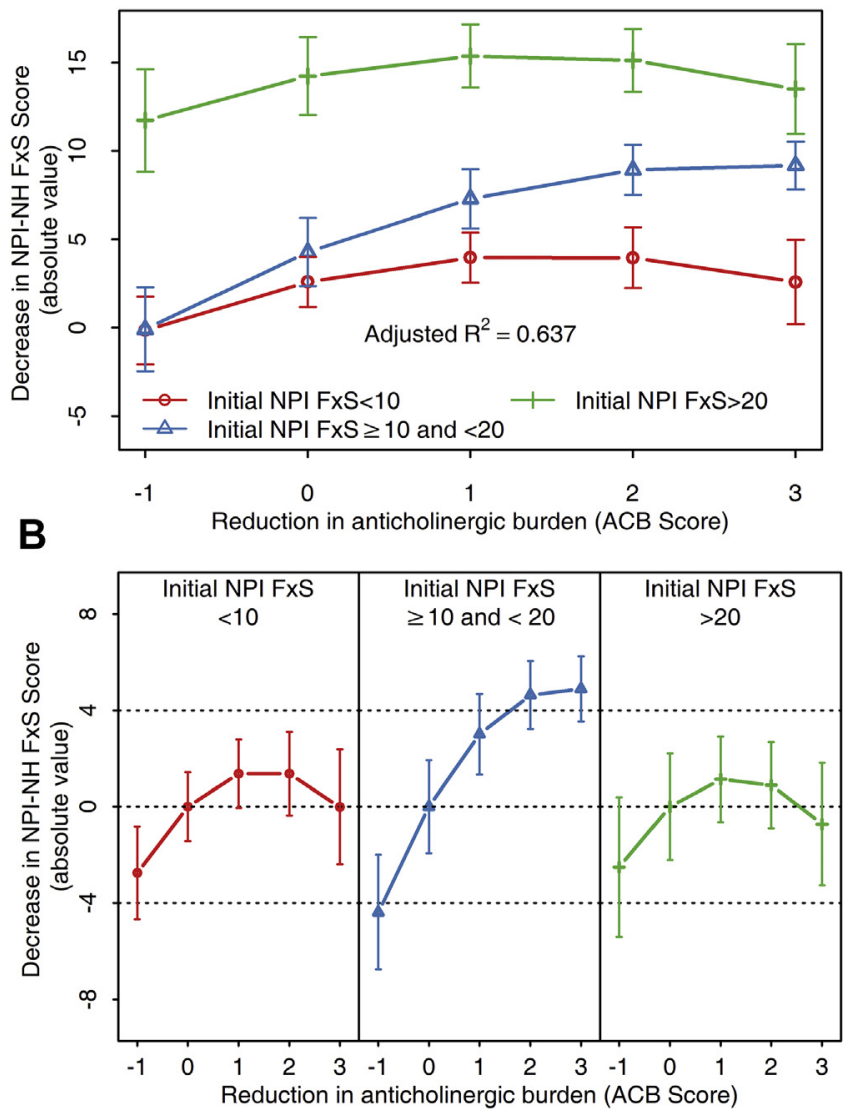

C
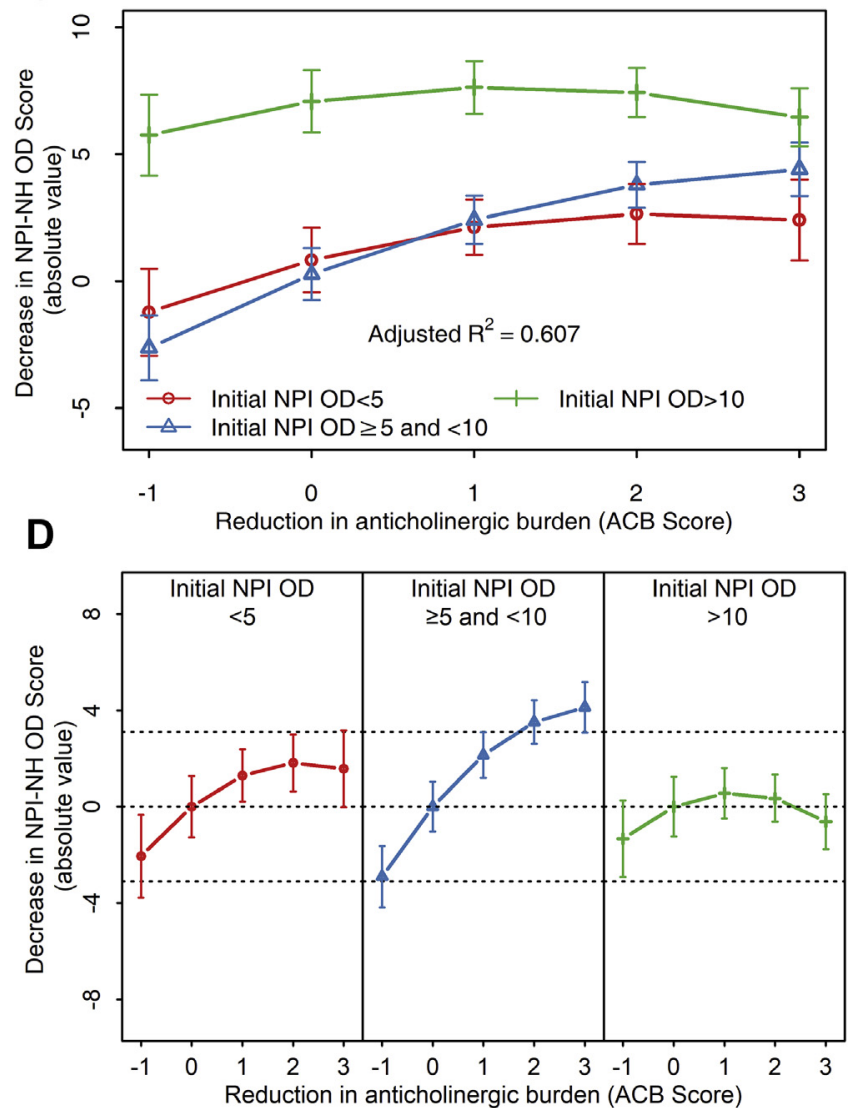

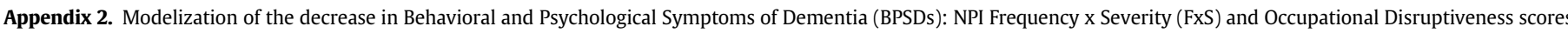
(OD) according to the reduction in anticholinergic burden as assessed by the Anticholinergic Cognitive Burden scale (ACB).

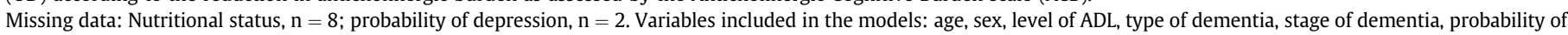
depression, level of comorbidities according to Charlson Comorbidity Index, nutritional status, and anticholinergic burden as assessed by the ACB.

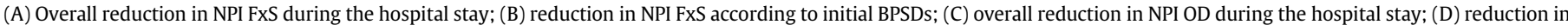
NPI OD according to initial BPSDs. 
A

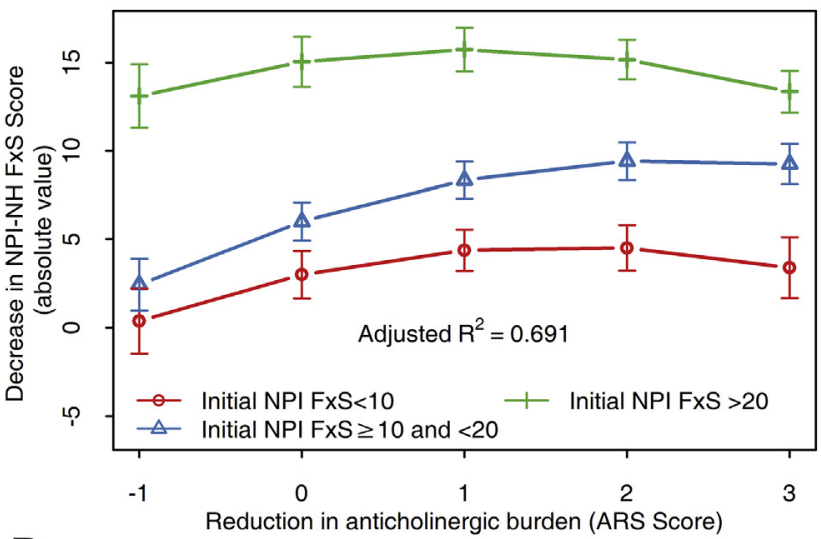

B

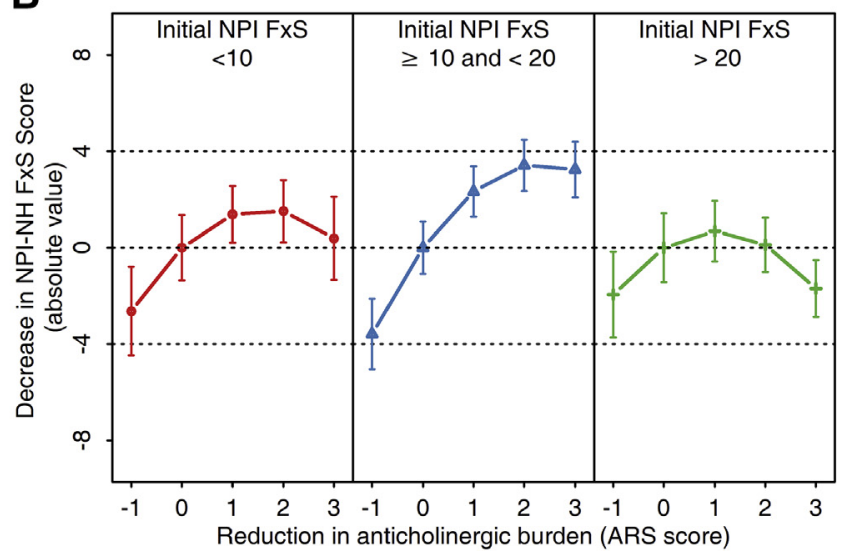

C

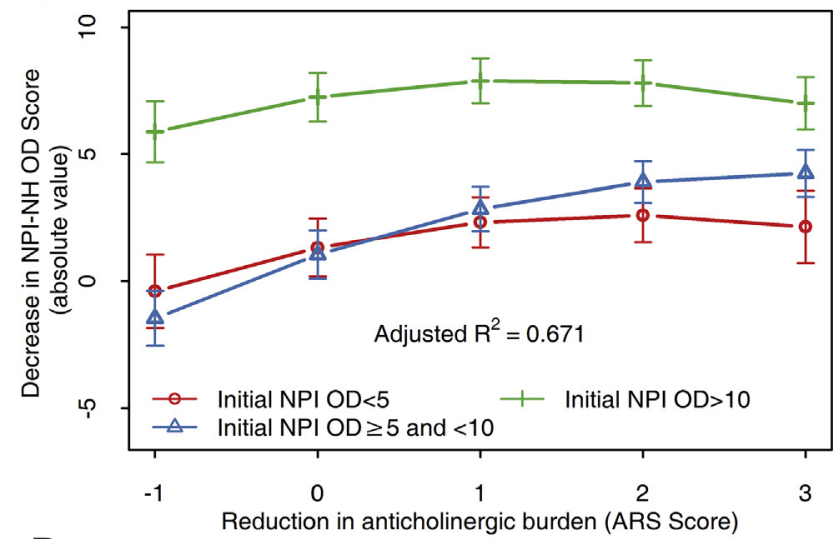

D

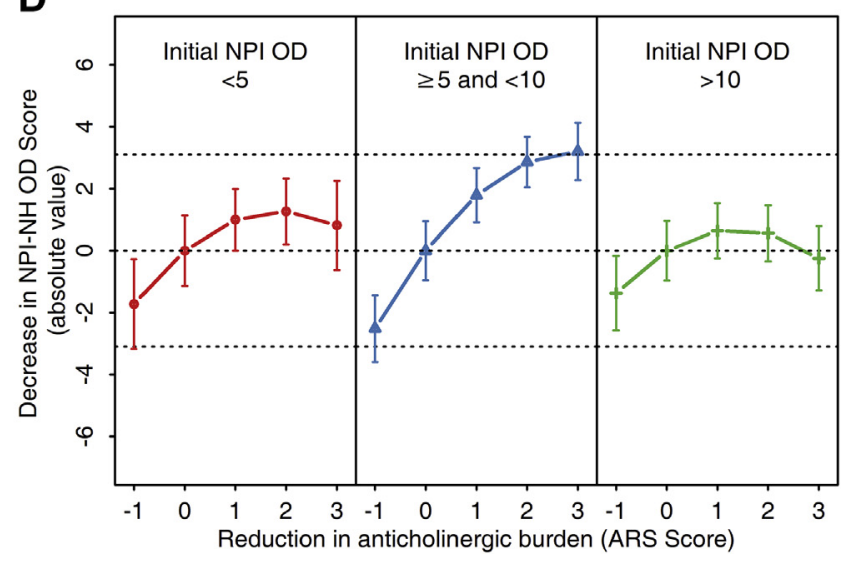

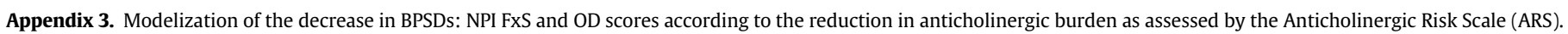

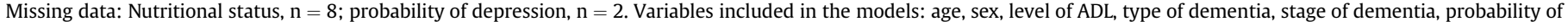
depression, level of comorbidities according to Charlson Comorbidity Index, nutritional status, and anticholinergic burden as assessed by the ARS.

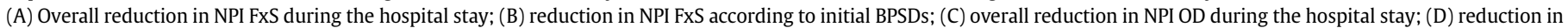
NPI OD according to initial BPSDs. 